\title{
Design and realization of a sensing module for machine tools status monitoring by multiple MEMS accelerometers integration
}

\author{
I-Chun Sun a, ${ }^{\text {, Jui-Min Tsai }}{ }^{\mathrm{a}}$, Kuo-Shen Chen ${ }^{\mathrm{a}}$, Yun-Hui Liu ${ }^{\mathrm{b}}$, Tian-Shiang Yang ${ }^{\mathrm{a}}$ and Mi-Ching Tsai ${ }^{\mathrm{a}}$ \\ a Department of Mechanical Engineering, National Cheng-Kung University, No.1 Daxue Rd., East Dist., Tainan City \\ 701, Taiwan \\ ${ }^{\mathrm{b}}$ Department of Mechanical Engineering, Southern Taiwan University of Science and Technology, No.1 Nantai St., \\ Yungkang Dist., Tainan City 710, Taiwan
}

*Corresponding Author: j803916811@gmail.com

\begin{abstract}
In modern Industry 4.0 era, status monitoring of machine tools is an important target and vibration monitoring is a commonly used way for machine tools life assessment. However, the vibration characteristics of machine tools are usually complicated and cover a very wide spectrum. Any accelerometers will suffer from its sensitivity-bandwidth limitation and high cost. As a result, an integrated vibration sensing module is proposed here by integrating various MEMS accelerometer chips. Through structure and circuit design, the vibration sensing module is integrated as well as the disadvantage, low sensitivity, of MEMS accelerometer is overcome. Furthermore, the module is then compared to a piezoelectric accelerometer as the standard for accessing the performance. Additionally, the vibration domain knowledge is also investigated and also applied to the machine tools for examining its feasibility. In the future, with more domain knowledge, it is expected that more sophisticated model would be developed for better predicting the machine status for enhancing the manufacturing reliability.
\end{abstract}

Keywords: MEMS accelerometer, Machine tools status monitoring, Vibration domain knowledge.

\section{Introduction}

Machine tools such as computer numerical control (CNC) milling machines play key roles in modern manufacturing industry and they usually represent the highest cost in the production process of the manufacturing industry besides raw materials ${ }^{(1)}$. The quality of machined products is largely depended on the status of machines in various aspects ${ }^{(2)}$. As a result, appropriate condition monitoring would be essential for both quality control and the safety and life assessment of machine tools especially for the current Industry $4.0 \mathrm{era}^{(3,4)}$. There are numerous states related to the healthy and life of typical CNC machine tools in various sub-systems ranged from driving motors, high speed spindles, lead screw and fixtures, and various cutting tools, as well as the cooling systems ${ }^{(5,6)}$. Among all these possible physical signals, machine vibration is particular meaningful for condition monitoring of machine tools, because the sources of vibration are quite complicate, such as gear meshing, bearing damage, shaft rotation imbalance, and chattering during cutting. Vibrations caused by these sub-systems have different time and frequency characteristics and usually distributed in a range around $10 \mathrm{kHz}$. Traditionally, piezoelectric accelerometers have been used for measuring vibration of machines ${ }^{(7)}$ for its high sensitivity and low noise level. However, a single piezoelectric accelerometer may not capable to faithfully measure the vibration of high speed machine tools due to its tradeoff between sensitivity and bandwidth. In addition, it is much more expensive and also more space-consuming then MEMS accelerometers if multiple piezoelectric accelerometers were hired.

Recently, with the advanced in microelectromechanical systems (MEMS) technology, MEMS accelerometers become an alternative choice in vibration measurement and they have been widely used in automobile and mobile communications. In the machine condition monitoring field, 2-axis MEMS accelerometers are also used to monitor rotating machine, such as motor ${ }^{(8)}$. Moreover, the design 
of 3-axis MEMS accelerometers have also been applied on robot $\operatorname{arm}^{(9)}$. However, although they are much cheaper and compact, their performances are usually worse than piezoelectric accelerometers and any single MEMS accelerometers also suffer from sensitivity-bandwidth tradeoff.

Despite of the above concerns, MEMS accelerometers are still attractive due to its low cost and miniaturization design, as well as their potential to be integrated with wireless network. In addition, individual MEMS accelerometers have exhibited acceptable performance in specific applications. Technically, it is possible to integrate several MEMS accelerometers together to resolve the tradeoff without changing the overall dimension significantly. As a result, the development of such a module of integrated MEMS accelerometers is a non-trivial task.

Based on our preliminary presentation in Chime Ball Technology Thesis Award ${ }^{(10)}$, this work is motivated by the above addressed needs and the ultimate goal is to develop a vibration sensing module by integrating MEMS accelerometers for monitoring the health condition of machine tool. Technically, there are three steps to achieve the final goal. First, several MEMS accelerometers and their associated amplification circuit must be integrated for optimizing the overall performance. Second, appropriated data reduction schemes should be coded in the associate firmware for effectively data processing to acquire feature index in both time and frequency domain. Moreover, the relation between feature index and cutting parameters would also be investigated and further applied to machine condition monitoring. Finally, these processed data could be uploaded to cloud via internet for accessing remotely. Meanwhile, by in cooperating with big data or AI technologies, machine condition diagnosis and life prediction could also be performed in the future.

As shown in Fig. 1, the remainder of this paper presents the investigation on realization the concept in detail. The design integrated MEMS accelerometers is addressed in Section II, followed by the realization and bandwidth testing results presented in Section III. Meanwhile, the investigation of vibration domain knowledge for machine tools is presented in Section IV and the demonstrations on CNC miller and robot arm are then addressed in Section $\mathrm{V}$ for validating the proposed concept. Finally, Section VI briefly discusses the current results, provides future perspective, and concludes this work.

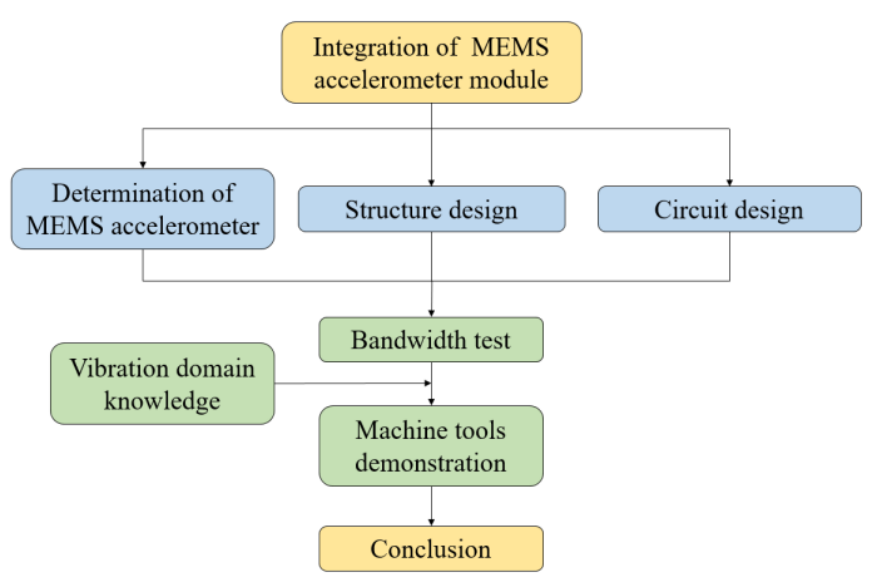

Fig. 1. The overall scope of this paper.

\section{Analysis and design of sensing module}

\subsection{Determination of MEMS accelerometers}

There are three types of MEMS accelerometers, which are shown in Fig. 2, selected in this work owing to their different dynamic characteristics ${ }^{(11)}$. For detecting high frequency vibration signal, the Analog Device ADXL001 single axis accelerometers is chosen for its wide bandwidth (up to $22 \mathrm{kHz}$ ). However, its sensitivity is only $25 \mathrm{mV} / \mathrm{g}$. Meanwhile, the dual axis MEMS accelerometer, ADXL203, is employed for medium frequency range. Its bandwidth is about $5.5 \mathrm{kHz}$ and sensitivity is $1000 \mathrm{mV} / \mathrm{g}$ for both axes. Finally, low frequency vibration signal is extracted by the tri-axial accelerometer, ADXL327. It has a relative low bandwidth $(1.6 \mathrm{kHz})$ and a moderated sensitivity $(420 \mathrm{mV} / \mathrm{g})$. As a result, three categories, high, middle and low bandwidth, are classify in this work, as shown in Fig. 3.

However, by means of integration accelerometers, the vibration signal is measured through total five accelerometers simultaneously. To integrate the results of three types accelerometers, Butterworth filters will be designed for processing signal as shown in Fig. 4 in the near future.

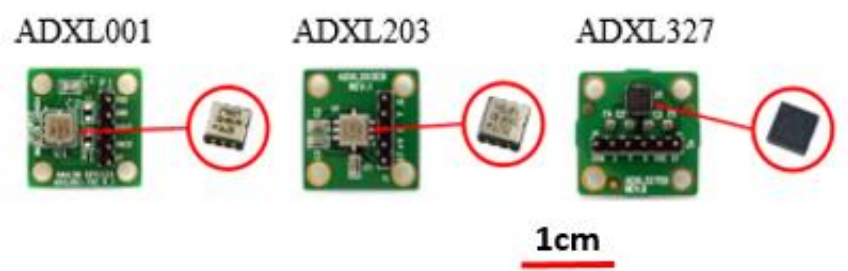

Fig. 2. MEMS accelerometers. 


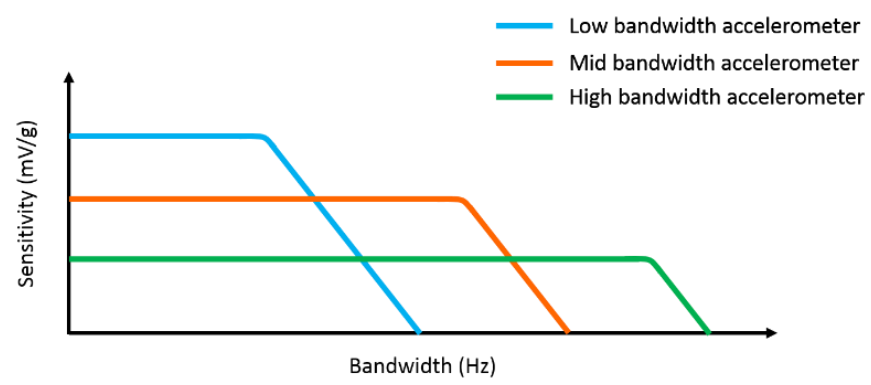

Fig. 3. Schematic plot of accelerometers integration.

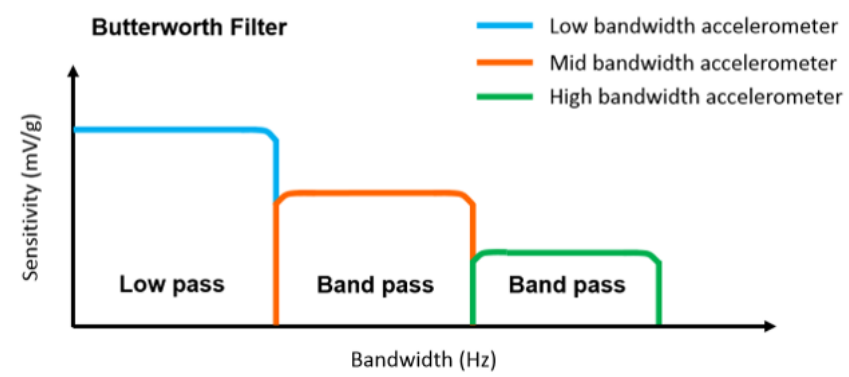

Fig. 4. Schematic plot of signal after Butterworth filter.

\subsection{Circuit layout and bandwidth testing}

As mentioned above, the sensitivity of ADXL001 is low. To improve the sensitivity, the amplifier circuit should be included in this integrated module. In addition, the output signal of MEMS accelerometer consists of a DC-biased AC voltage. As a consequence, the DC bias should be removed first in order to achieve a lager amplification before saturation. For conducting the above mentioned goals, the OP-AMP based circuit is employed. The schematic and layout of the circuit is shown in Fig. 5. and Fig. 6 Additionally, to ensure the circuit would not influence signal, it is necessary to measure the bandwidth of the circuit. A scenario signal is then provided by a function generator (Tektronix, AFG3021). For covering MEMS accelerometer bandwidth, a sine signal from $10 \mathrm{~Hz}$ to $10 \mathrm{kHz}$ with an amplitude of $20 \mathrm{mV}$ is input.

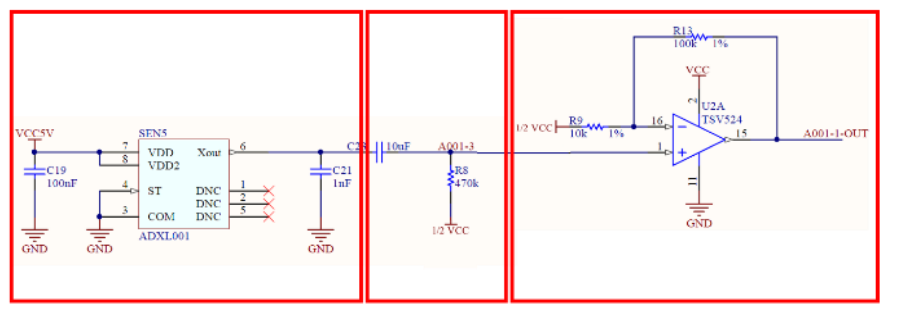

ADXL001 Circuit

High pass filter

Noninverting amplifier

Fig. 5. Schematic plot of OP-AMP circuit.

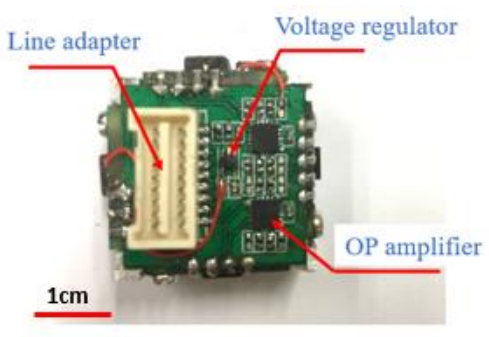

Fig. 6. Layout of OP-AMP circuit.

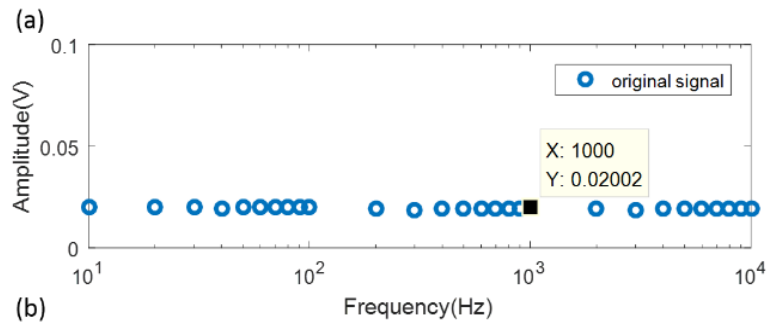

(b)

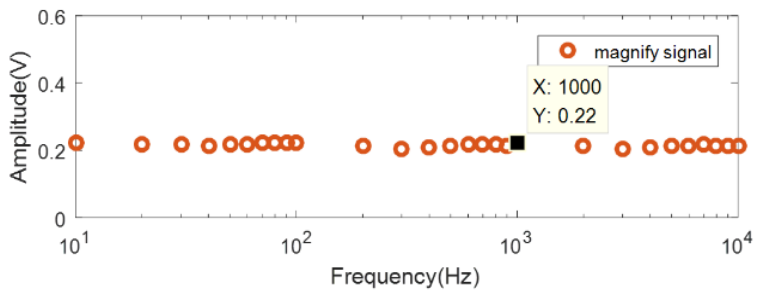

Fig. 7. The frequency response of the amplification circuit.

The output signal, as shown in Fig. 7, is amplified by a ratio of 11 and amplification ratio is essentially constant for less than $10 \mathrm{kHz}$, which is sufficient for MEMS accelerometer bandwidth. As a result, this amplified circuit can be treated as a pure amplifier in this module.

\section{Realization and functional assessment}

\subsection{System integration}

To shrink the size of the designed module as much as possible for applying to machine tools, five MEMS accelerometers and the amplification circuit are integrated into one adaptor. Meanwhile, the signal output wires are also integrated into single output wire. The adaptor, as shown in Fig. 8, is designed as the platform for adapting all MEMS accelerometers and their associate electronic. In addition, since ADXL001 is a single axis sensor, three orthogonal surface should be used to place them. On the other hand, ADXL203 and ADXL327 can be mounted on any surface and the remaining surfaces can be used for placing necessary electronics and line adapter. Finally, total size of this module is reduced to $13 \mathrm{~mm} \times 22 \mathrm{~mm} \times 22 \mathrm{~mm}$, which is small enough for the purpose of installing inside the spindle of machine tools. 

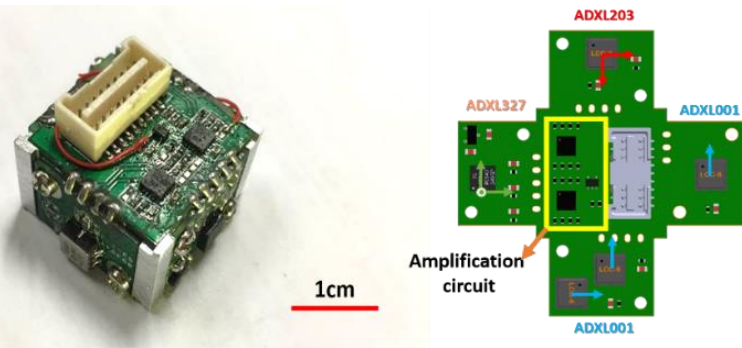

Fig. 8. The integration module and its schematic plot.

\subsection{Module bandwidth testing}

After module designed, the bandwidth of the whole module should be measured to make sure it is sufficient for extracting vibration signal of machine tools. The bandwidth testing system is as shown in Fig. 9. The designed module and a referenced standard sensor, piezoelectric accelerometer (PCB, 354C03), are screwed in the same reference surface on the shaker (TIRA, TV51120), which is command by a sweep sine signal to examine the bandwidth of these MEMS accelerometers. Besides, the output signals of accelerometers are captured by a spectrum analyzer (B\&K type 3560C) for measuring their frequency characteristic. The results are as shown in Fig. 10 and Table 1. The bandwidth testing results., which is good enough to extract some failure signal of machine tools, such as chattering signal (about $2 \mathrm{kHz}$ ).

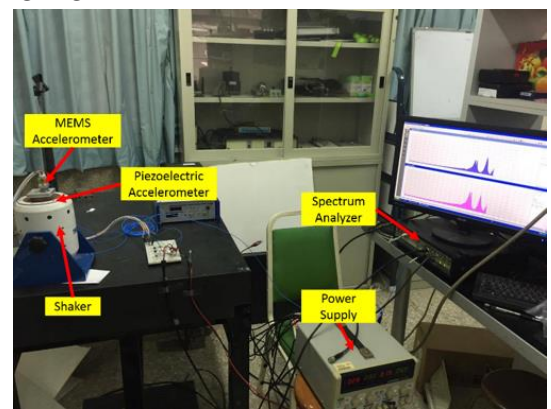

Fig. 9. The bandwidth testing system.

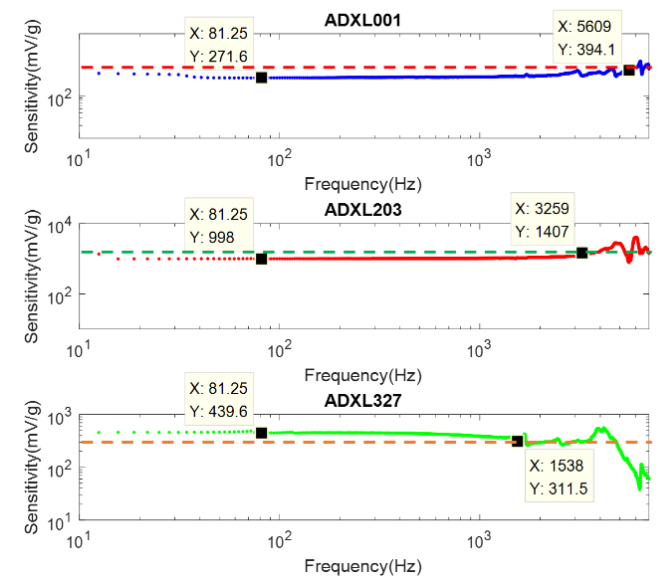

Fig. 10. The frequency response of three MEMS accelerometers.
Table 1. The bandwidth testing results.

\begin{tabular}{|c|c|c|}
\hline Type & Sensitivity $(\mathrm{mV} / \mathrm{g})$ & Bandwidth $(\mathrm{Hz})$ \\
\hline ADXL001 & 271 & 5609 \\
\hline ADXL203 X & 998 & 3259 \\
\hline ADXL327 X & 439 & 1538 \\
\hline
\end{tabular}

\section{Develop vibration domain knowledge}

Through the effort of module design and functional test, an integrated MEMS accelerometer is developed. For applying to machine tools vibration monitoring, the next issue is to develop vibration domain knowledge for machine tools through feature extraction. To develop vibration domain knowledge there are a few tasks should be accomplished, including data acquisition setup, feature extraction and data base establishment. The initial effort toward these issues are going to be performed and addressed.

\subsection{Data acquisition system setup}

First, the vibration signal is collected from a PMC 5-axis CNC miller as shown in Fig. 11. To measure the vibration of machine tools during operating a kistler 3 -axis accelerometer (kistler, 8763BB) is fixed on the spindle. Meanwhile, a single axis accelerometer is mounted on the platform in order to collect vibration signal from raw material. Second, the vibration signal is then acquired by data acquisition system (NI-9215) with a sampling rate of $40 \mathrm{kHz}$. Finally, to assess various feature measurement from vibration signal, the signal is then extracted by a constructed program via LabVIEW, which analyzes the signal in time and frequency domain automatically.

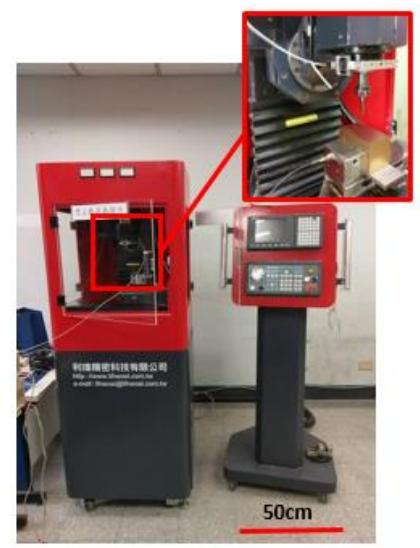

Fig. 11. The experiment system of machine vibration monitoring. 


\subsection{Feature extraction}

Through the effort of establishing data acquisition system, we can proceed data reduction part. The purpose of feature extraction is to reduce the dimension of the raw data from signal but at the same time maintain the relevant information about health condition of machine tools. In this research, statistically measurements of vibration signal are taken for both time and frequency domain methods. For time domain characteristics, RMS and Kurtosis are calculated. Meanwhile, by performing fast fourier transform (FFT), the key frequency characteristics can also be identified. Both measurement have significant implications in machine tools monitoring. For example, RMS represents the overall vibration level. In addition, Kurtosis is four central moments and commonly used in machine condition monitoring, because failures of machine components often excite relatively large pulses in the time domain waveform. On the other hand, vibration spectrum can be considered as the vibration signature of machines, since frequency characteristics are somewhat different in different condition and some spectrum can be linked to operating parameters of machine tool ${ }^{(12)}$. Spindle rotating speed frequency and chattering frequency will be identified in the following experiments.

\subsection{Data base establishment}

To acquire the characteristics of vibration signal during cutting in different cutting parameters, the rotating speed is varied from 7000 to $10000 \mathrm{rpm}$. Meanwhile, the depth of cut is adjusted from 0 to $0.4 \mathrm{~mm}$. Besides, to obtain larger change of vibration level without cutting too much material, copper is chosen as material in this cutting experiment. After data acquisition and reduction, the characteristics of above signal processing methods are provided. As shown in Fig. 12, the RMS level of spindle vibration increases with the rotating speed as well as depth of cut. For Kurtosis method, as shown in Fig. 13, the Kurtosis level is about 3 for low cutting depth regardless of different rotating speed. As cutting depth increases, Kurtosis value will drastically increase. However, there is no too much change at platform.

For frequency domain results, the raw data in time domain from above experiments are transformed into frequency domain via FFT. Some characteristic frequency will be identified, including spindle rotating speed frequency and chattering frequency.
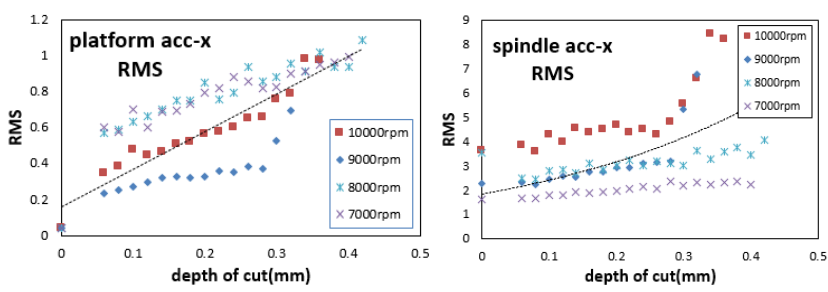

Fig. 12. RMS results of platform and spindle vibration.
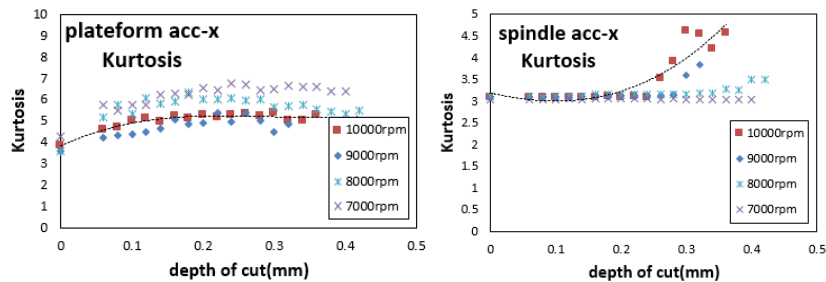

Fig. 13. Kurtosis results of platform and spindle vibration.
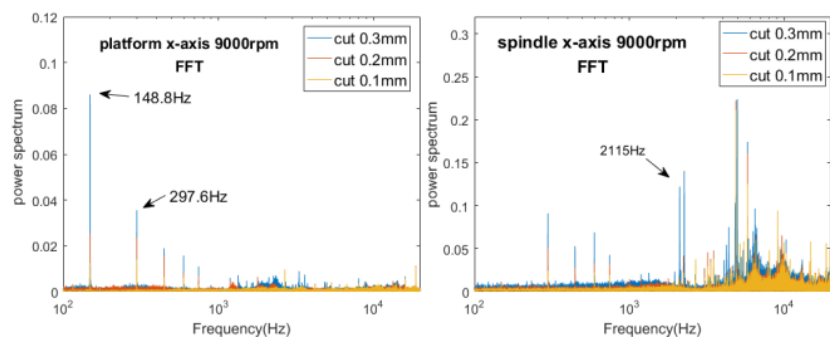

Fig. 14. FFT results of platform and spindle vibration.

Take 9000rpm results as example, as shown in Fig. 14, the spindle rotating frequency at about $150 \mathrm{~Hz}$ as well as its harmonics can be found out and it is also the major component of vibration spectrum at platform. On the other hand, the chattering signal at about $2 \mathrm{kHz}$ can be obviously identified in the case of large cutting depth at spindle.

In summary, the relation between cutting parameters and the feature indexes of vibration signal whether in time or frequency domain is developed in this section. The results will be applied to the developed module for examining the feasibility of the module by comparing with reference accelerometer.

\section{Demonstration and application}

The developed module is then applied to PMC 5-axis CNC miller and robot arm for demonstrating its possible applications in monitoring machines. PMC 5-axis CNC miller is used for examining vibration signal monitoring. On the other hand, an old robot arm with abnormal noise is employed for demonstrating the capability of machine health condition monitoring. Meanwhile, the Wi-Fi DAQ 
and IP camera are also implemented in robot arm monitoring experiment to demonstrate the feasibility of remote monitoring.

\subsection{PMC 5-axis CNC miller vibration monitoring}

For measuring the vibration of spindle, the developed module and a piezoelectric accelerometer (kistler, 8763BB), served as reference, are mounted on the spindle of the miller. As mentioned previously, vibration signal from both the developed module and the kistler accelerometer will be further processed in time and frequency domain. The CNC is commanded by 3000rpm rotating speed and 0.02 to $0.14 \mathrm{~mm}$ cutting depth. Then the experiment similar with last section will be repeated. First, for time domain, the RMS values of vibration signals are calculated. As shown in Fig. 16, the results show that RMS of the developed module are close to the results of reference accelerometer and also indicate the vibration level increases with rotating speed as mentioned in last section.

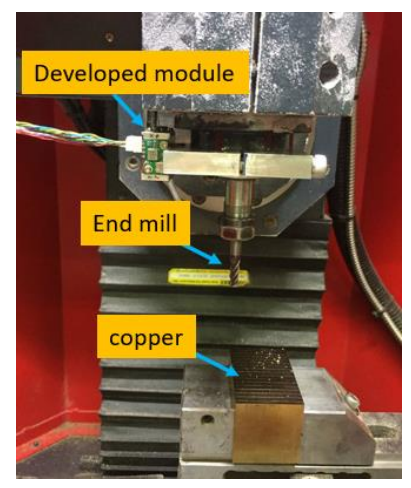

Fig. 15. The setup of CNC miller vibration monitoring
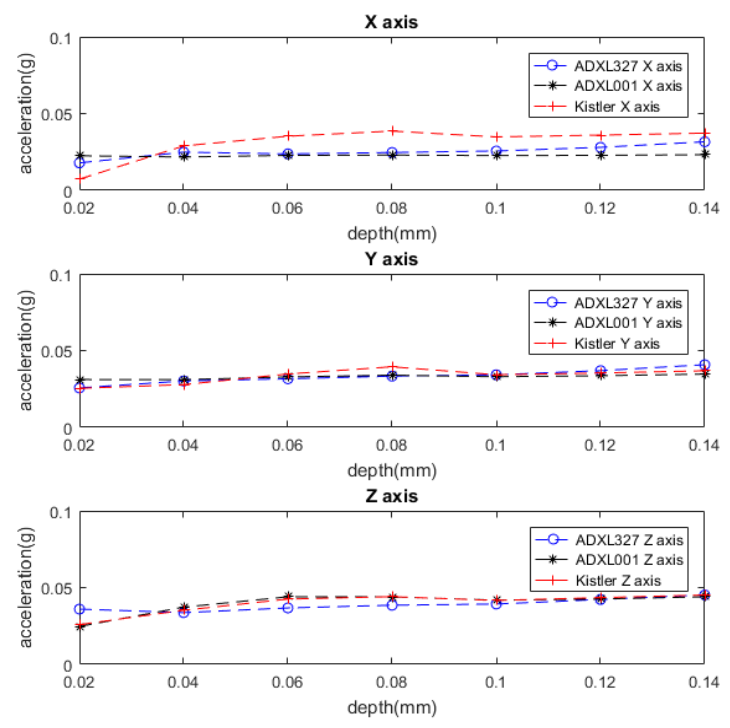

Fig. 16. The time domain results of $\mathrm{CNC}$ miller monitoring experiment.
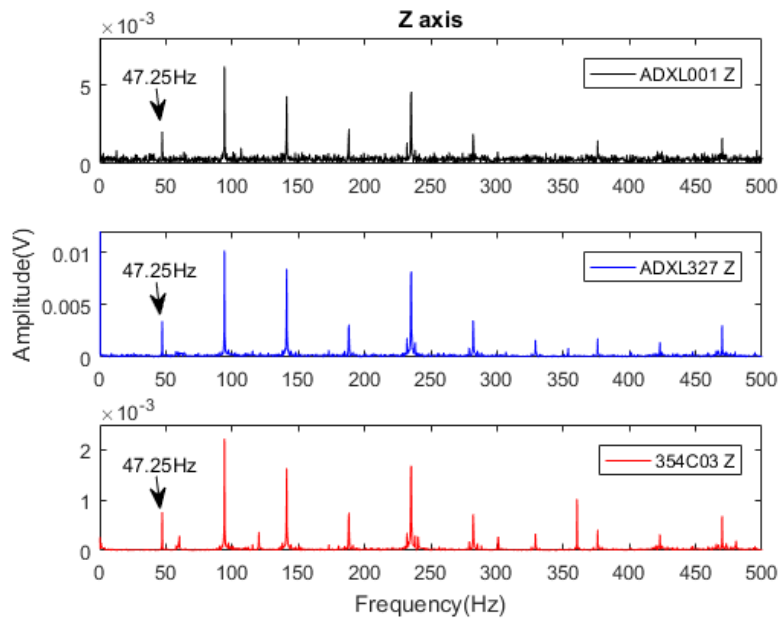

Fig. 17. The frequency domain results of CNC miller monitoring experiment.

On the other hand, for frequency domain, both accelerometers can successfully extract the rotating speed (about $47.25 \mathrm{~Hz}$ ) and its harmonics as shown in Fig. 17. As a result, this developed module not only can obtain the similar results with the piezoelectric accelerometer, but also shrinks its size.

\subsection{Health condition monitoring of robot arm}

In addition to vibration sensing on the $\mathrm{CNC}$ miller, to demonstrate its capability for machine tools health condition monitoring, the developed module is also applied on a old robot arm with some abnormal noise from the sixth joint at some posture. As shown in Fig. 18, this module is mounted on the sixth joint of the robot arm and the vibration signals are captured by DAQ and LabVIEW.

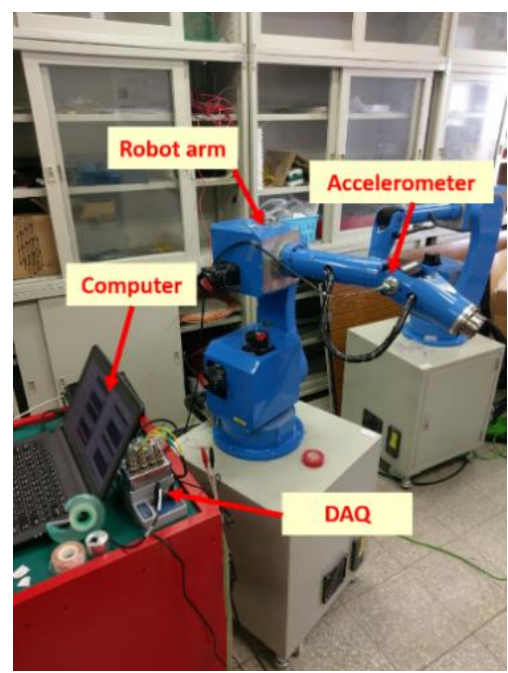

Fig. 18. The robot arm monitoring system. 
To make the robot arm produce noise, the posture of robot is continually adjusted. Meanwhile, the vibration signal is extracted by the developed module as noise sound arise. The time domain result is as shown in Fig. 19. The abnormal noise is arise from about 6 to 14 second and the vibration level also increases obviously. Last but not least, these abnormal vibration signals are also transformed to frequency domain by FFT. We can identify a $304 \mathrm{~Hz}$ signal and its harmonics from power spectrum of any MEMS accelerometer as shown in Fig. 20.

Meanwhile, the Wi-Fi DAQ and IP camera are also implemented in this experiment to demonstrate the feasibility of remote monitoring. As shown in left hand side of Fig. 21, the IP camera allows instant imagery monitoring of machines via internet. By in cooperating with Wi-Fi DAQ and LabVIEW, the vibration data can also be viewed remotely.

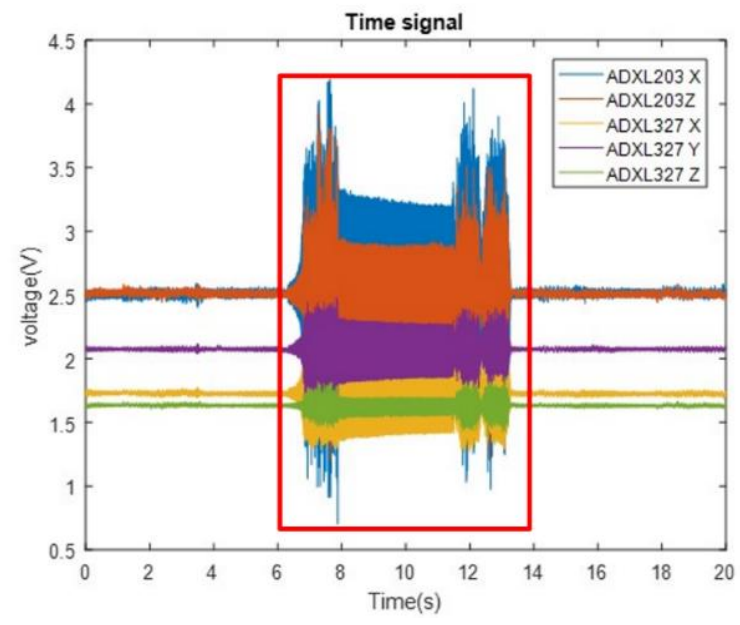

Fig. 19. The time domain results of robot arm.

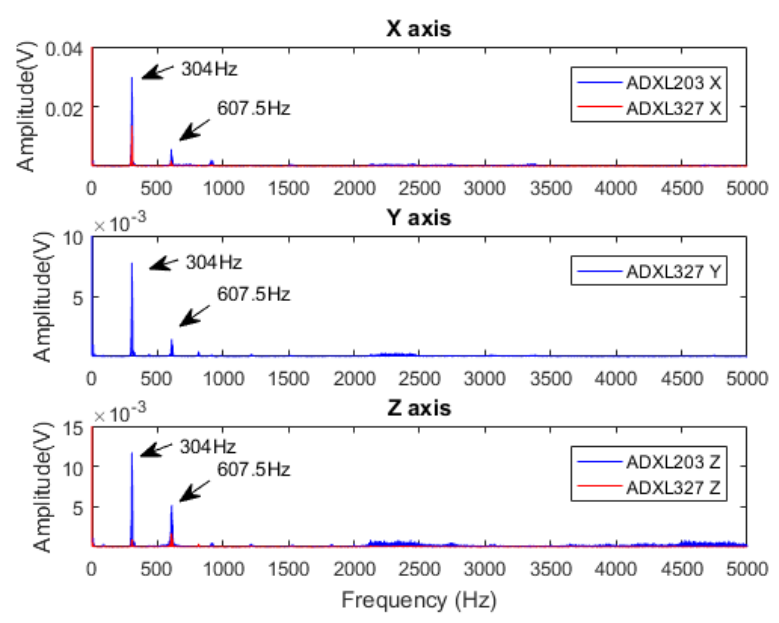

Fig. 20. The frequency domain results of robot arm.

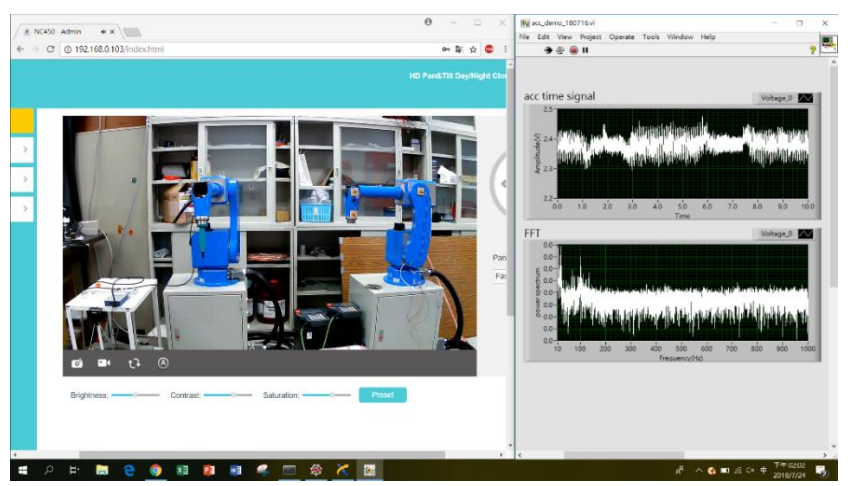

Fig. 21. The remote monitoring system.

\section{Conclusion and future works}

Machine tools play key roles in modern manufacturing industry. The status monitoring becomes essential for product quality insurance and cost saving. We focus on the vibration monitoring of machine tools in this work, so size and cost of the sensor are key issues in vibration detecting. In addition, development of vibration domain knowledge is also important to accomplish condition diagnosis. This work presents our initial effort for conducting the above mentioned goals. For the purpose of low cost monitoring, a vibration sensing module which is integrated by several MEMS accelerometers with different dynamic performance is proposed and investigated. Furthermore, to test the bandwidth of this module and check whether it is sufficient for vibration monitoring of machine tools, the developed module is compared with a reference piezoelectric accelerometer by mounted on shaker. The primary test results on the dynamic performance characteristics indicate that those MEMS accelerometers are potentially capable for measuring vibration of machine tools. In addition, the preliminary study toward vibration domain knowledge in both time and frequency domain is performed and addressed. Finally, the developed module is used on a CNC miller and an old robot arm for examining the feasibility of the developed module by monitoring the operating status of multiple machines.

In the near future, the size of the module could be further shrink by circuit miniaturization and reducing the size of the adapter. Moreover, as the advanced in MEMS technology, MEMS accelerometer with better dynamic performance could be chosen. For condition diagnosis, due to the source of vibration signal is pretty complicated, analytical neural network will be applied and the model will be trained by the features extracted from accelerometer in both time and frequency domain. It is expected that a 
more suitable model based on ANN would be developed for better describing and predicting the machine status for enhancing the manufacturing reliability.

\section{Acknowledgment}

This paper is supported by the ministry of science and technology (MOST) of Taiwan under the contract no. 105-2218-E-006-015, 107-2218-E-006-002, 107-2221-E218-029 and 107-2622-8-006-015. The supports and guidance from the PI: Prof. S. J. Huang and others co-PIs, as well as the lab manager, Miss $\mathrm{H}$. Cheng, are greatly appreciated.

\section{References}

(1) S. Kalpakjian and S. R. Schmid, Manufacturing Process for Engineering Materials, Prentice Hall, 2008

(2) P. Stavropoulos, D. Chantzis, C. Doukas, A. Papacharalampop and G. Chryssolouris, "Monitoring and Control of Manufacturing Processes: a Review," Procedia CIRP, Vol. 8, pp. 421-425, 2013

(3) M. Rüßmann, M. Lorenz, P. Gerbert, M. Waldner, J. Justus, P. Engel, and M. Harnisch, "Industry 4.0: The Future of Productivity and Growth in Manufacturing Industries," Boston Consulting Group, 2015

(4) H. Lasi. Heiner, et al. "Industry 4.0," Business \& Information Systems Engineering, Vol. 6, pp. 239-242, 2014

(5) P. Prickett and C. Johns, "An Overview of Approaches to End Milling Tool Monitoring," International Journal of Machine Tools and Manufacture, Vol. 39, pp.105-122, 1999

(6) R. Teti, K. Jemielniak, G. O’Donnell and D. Dornfeld, "Advanced Monitoring of Machining Operations," CIRP Annals-Manufacturing Technology, Vol. 59, pp. 717-739, 2010

(7) J.K. Sinha, K. Elbhbah, "A future possibility of vibration based condition monitoring of rotating machines," Mechanical Systems and Signal Processing, Vol. 34, pp. 231-240, 2013

(8) S. B. Chaudhury, M. Sengupta, K. Mukherjee, "Vibration Monitoring of Rotating Machines Using MEMS Accelerometer," International Journal of Scientific Engineering and Research, Vol. 2, pp. 5-11, 2014

(9) A. A. Jaber and R. Bicker, "Design of a Wireless Sensor Node for Vibration Monitoring of Industrial Machinery," International Journal of Electrical and
Computer Engineering, Vol. 6, pp. 639-653, 2016

(10) I. C. Sun, J. M. Tsai and K. S. Chen, "Design and realization of a sensing module for machine tools status monitoring by multiple MEMS accelerometers integration," Taiwan Precision Technology Workshop, 2018.

(11) Analog Devices website, "http: //www. analog.com/ en/index.html"

(12) S. S. Rao and F. F. Yap, Mechanical vibrations, Vol. 4. Upper Saddle River: Prentice Hall, 2011 